\title{
Erratum to: Dose-related effect of statins in patients with endovascular coiling or microsurgical clipping for aneurysmal subarachnoid hemorrhage: updated study-level meta-analysis
}

Kyu-Sun Choi ${ }^{1}$. Jae Min Kim ${ }^{1} \cdot$ Hyeong-Joong $\mathrm{Yi}^{1} \cdot$ Seon-Heui Lee ${ }^{2}$.

Taeho $\mathrm{Lim}^{3}$ • Wonhee $\mathrm{Kim}^{4} \cdot$ Youngsuk Cho ${ }^{4} \cdot$ Jin-Hwan Cheong ${ }^{1}$

Published online: 31 July 2017

(C) Springer-Verlag GmbH Germany 2017

Erratum to: Eur J Clin Pharmacol

DOI 10.1007/s00228-017-2221-7

The original publication of the paper contains an error.

The correct grant number should be HC15C3401.

The online version of the original article can be found at http://dx.doi.org/ 10.1007/s00228-017-2221-7

Seon-Heui Lee

sunarea87@gachon.ac.kr

1 Department of Neurosurgery, College of Medicine, Hanyang University, Seoul, South Korea

2 Department of Nursing Science, College of Nursing, Gachon University, 191 Hambakmoero, Yeonsu-gu, Incheon 406-799, South Korea

3 Department of Emergency Medicine, College ofMedicine, Hanyang University, Seoul, South Korea

4 Department of Emergency Medicine, College of Medicine, Hallym University, Seoul, South Korea 